\title{
Sleep pattern in epilepsy patients: a polysomnographic study
}

\author{
Mohamed A. Tork', Hebatallah R. Rashed', Lobna Elnabil ', Nahed Salah-Eldin', Naglaa Elkhayat', \\ Ayman A. Abdelhady ${ }^{1}$, M. Ossama Abdulghani ${ }^{1}$ and Khaled O. Abdulghani ${ }^{2^{*}}$ (i)
}

\begin{abstract}
Background: Sleep disorders and epilepsy commonly exist and affect each other. Patients with epilepsy often complain of poor sleep and on the other hand, poor sleep makes epilepsy control difficult.

Objectives: We aimed at comparing the sleep disturbances in a group of patients with medically controlled epilepsy versus another group with medically refractory epilepsy, from the electrophysiological standpoint.

Subjects and methods: Sixty epilepsy patients were included; half of them with controlled epilepsy were assigned as group I, and the other half with refractory epilepsy was assigned as group II. All patients had an overnight polysomnogram and sleep EEG done. We excluded any patient with abnormal general or neurological clinical examination.

Results: Patients in group II, had significantly delayed sleep onset latency and REM latency. However, higher arousal index, insomnia, and periodic limb movement index were found to be significantly higher in group I. Respiratory events; as light sleep durations, were observed to be higher in Group II, in addition to apnea-hypopnea index that was significantly higher in this group.
\end{abstract}

Conclusion: Epilepsy affects sleep architecture and sleep-related events. Patients with refractory epilepsy suffer from more disturbance in sleep patterns. Moreover, antiepileptic drugs can have a diverse effect on sleep architecture and quality in epileptic patients.

Keywords: Refractory epilepsy, Sleep pattern, Polysomnography

\section{Introduction}

Sleep disorders and epilepsy commonly exist and affect each other. Patients with epilepsy often complain of poor sleep, and on the other hand, poor sleep makes epilepsy control difficult [1]. Treatment of sleep disorders improves quality of life of patients with epilepsy [2]. In the present study, we aimed at comparing the sleep disturbances in a group of patients with medically controlled epilepsy versus another group with medically refractory epilepsy, from the electrophysiological standpoint.

\section{Subjects and methods}

Sixty adult epileptic patients were enrolled in this crosssectional study from Ain Shams University Hospitals,

\footnotetext{
* Correspondence: Khaled.abdulghani@med.helwan.edu.eg ${ }^{2}$ Departemnt of Neurology and Psychiatry, Helwan University, School of Medicine, Helwan, Egypt

Full list of author information is available at the end of the article
}

Epilepsy Clinic. All subjects signed a written informed consent before participating in any the study. Enrolled patients aged between 18 and 57 years of age, and the diagnosis of epilepsy was made according to the International League Against Epilepsy (ILAE) classification and terminology [3]. We excluded any patient with abnormal general or neurological clinical examination.

Subjects included were divided into two groups. Thirty patients were controlled on medical treatment with no or one seizure/month and were assigned as group I. The other thirty patients diagnosed as refractory epilepsy, defined as persistence of seizures, more than one seizure a month after trials of at least two antiepileptic drugs (AEDs) with maximally tolerated doses sequentially or in combination, and duration of AED treatment of at least 2 years, were assigned as group II [4].

We excluded patients having seizures secondary to any other medical condition. In addition, we excluded 
patients with medical illnesses that possibly affect their sleep pattern (as hepatic and thyroid disorders). Moreover, we did not enroll patients with history of obstructive sleep apnea (OSA) prior to their epilepsy diagnosis, those with history of hypnotics or sedative intake, and those who recently discontinued their AEDs. Patients with narcolepsy and any other primary sleep disorder that require intervention with medication and potentially affect the results of the study were also excluded.

Subjects were able to withdraw voluntarily from the study at any time. Development of a clinically relevant change in medical condition, consent withdrawal, and difficulties of transportation as participation required an overnight stay in the sleep laboratory, were all reasons of patient's withdrawal.

All patients were subjected to full general and neurological examination including age, sex, and body mass index (BMI). Full epilepsy history including epilepsy type, seizure type(s) and frequency, and AEDs.

Ascertaining sleep and wake patterns, nighttime behaviors and sleep disorder symptoms, pertinent medical history, and medications were determined through subject interview. All subjects completed the sleep questionnaire "sleep history through structured sheet for sleep disorders center questionnaire" for the screening of sleep disorders [5].

Subjects underwent an overnight polysomnography (PSG) with electroencephalography (EEG) recording for at least 6 h, using a Nihon Kohden Neurofax-1200 EEG/PSG system (Tokyo, Japan). We used 18 channels for EEG, 2 channels for electrooculography, 4 channels for legs, and 1 channel for each chin electromyography. We also used nasal pressure transducer and naso-oral thermistor to monitor airflow and piezoelectric belts for thoracic and abdominal efforts, in addition to ECG, pulse oximetry, snoring, body position, and video recordings. We performed sleep staging and event scoring according to the American Association of Sleep Medicine Scoring Manual [6]. We traced apneas and hypopneas to derive the apnea-hypopnea index (AHI), defined as the number of respiratory events per sleep hour. Patients having an AHI $>5$ were considered to have sleep apnea [7].

The sleep parameters that were obtained by the PSG included (a) sleep latency and continuity measures (number of awakening more than 2 min duration, sleep efficiency, and number of arousal); (b) Sleep architecture including breathing events (AHI), and movement analysis and periodic limb movement (PLM) index with arousal, with no arousal, and total PLM index; and (c) rapid eye movement (REM) sleep analysis including REM\%, REM latency, and REM periods.

\section{Statistical analysis}

Data were collected, coded, and entered to a personal computer, IBM compatible. The data were analyzed with the SPSS version 11.0.1 (Statistical Package for Social Science, Chicago, USA). Descriptive statistics was displayed as mean \pm standard deviation (SD) for continuous data. Student $t$ test used for comparison of parametric data between the independent groups, Pearson correlation coefficient $(r)$ test to indicate correlation between different variables, and Chi-square test for comparison of qualitative data. The level of statistical significance $(P$ value) was set at 0.05 .

\section{Results}

The demographic data for both groups are shown in Table 1. There were no significant age or gender differences between medically refractory or controlled epilepsy patients. In addition, there was no significant difference between both groups as regards gender distribution and BMI.

Sleep efficiency was decreased in both groups; however, the difference between both groups was not statistically significant $(P>0.05)$. Patients in group II (refractory epilepsy), took longer time to sleep as indicated by delayed sleep onset latency, compared to controlled epilepsy group, which was statistically significant. On the other hand, arousal index was found to be higher in medically controlled epilepsy, as well as number of awakenings, and limb movement index which was statistically significant (Table 2).

Respiratory events were observed more frequently in group II, as AHI was higher among patients with refractory epilepsy compared to those with medically controlled epilepsy, although not reaching statistical significance. Light sleep (N1 and N2) was prolonged in both groups, but still being higher in group II, which was statistically significant. Deep sleep (N3) was decreased in both groups, but differences between both groups was not statistically significant (Table 3 ).

REM sleep was significantly decreased in group II compared to group I. REM latency was found to be longer in the group with refractory epilepsy, as compared to those with medically controlled epilepsy, but it was found to be statistically insignificant (Table 4).

Sleep symptoms including insomnia, excessive daytime somnolence (EDS), and snoring were reported in both groups. Patients with medically controlled epilepsy reported

Table 1 Comparison between both groups regarding demographic data

\begin{tabular}{llll}
\hline Variable & Group I & Group I & $P$ value \\
\hline Age of patients (years) & $29.1 \pm 10.8$ & $25.1 \pm 7.3$ & 0.1 \\
Male $(n)$ & $12(40 \%)$ & $14(46.7 \%)$ & 0.6 \\
Female $(n)$ & $18(60 \%)$ & $16(53.3 \%)$ & 0.6 \\
BMI (mean \pm SD) & $25.86 \pm 4.9$ & $26.2 \pm 5$ & 0.7 \\
\hline
\end{tabular}

$B M I$ body mass index, $n$ number 
Table 2 Comparison between both groups regarding Sleep Latency and continuity measures

\begin{tabular}{llll}
\hline Variable & $\begin{array}{l}\text { Group I } \\
\text { Mean } \pm \text { SD }\end{array}$ & Group I & $P$ value \\
\hline Sleep efficiency (\%) & $85.3 \pm 16.8$ & $83.56 \pm 14.1$ & 0.67 \\
Sleep onset latency (min) & $12.5 \pm 11.7$ & $23.89 \pm 31.5$ & 0.04 \\
Arousal index & $19.8 \pm 25$ & $10.2 \pm 9.3$ & 0.06 \\
Number of awakenings & $7.3 \pm 7.9$ & $6 \pm 5.8$ & 0.47 \\
PLM index & $3 \pm 5$ & $0.5 \pm 1.1$ & 0.01 \\
\hline
\end{tabular}

PLM periodic limb movement

insomnia more frequently than EDS and snoring, as compared to refractory epilepsy patients, and this was statistically significant. On the other hand, snoring and EDS were reported more frequently in patients with refractory epilepsy as compared to those with medically controlled epilepsy, and this was also statistically significant (Table 5).

As regards the seizure type, in group I "medically controlled epilepsy", there were 25 patients (83.3\%) with generalized tonic clonic seizures (GTC) and 5 patients (16.7\%) with focal seizures, whereas in group II "refractory epilepsy", there were 16 patients $(53.3 \%)$ with focal seizures and 14 patients $(46.7 \%)$ with generalized epilepsy. Thus, refractory epilepsy patients tend to have focal epilepsies than those of medically controlled epilepsy, and this was significant. Compared with medically controlled group with focal epilepsy, more patients with refractory epilepsy were having more temporal lobe seizures, and again, this was statistically significant.

As expected, patients with refractory epilepsy were taking more AEDs compared with those with medically controlled epilepsy, yet with no statistical significance. Most of the patients of group I were controlled on monotherapy, with 23 patients (76.7\%) taking first generation AEDs, and 7 patients (23.3\%) taking second generation AEDs. Patients in group II were maintained on polytherapy; all of them were taking first generation AEDs, and 27 patients (90\%) were combining first- and second-generation AEDs. Patients on valproic acid (VPA) and levetiracetam (LEV) were found to be significantly more in the refractory epilepsy group as compared to medically controlled epilepsy patients. On the other hand, patients on carbamazepine (CBZ), phenytoin

Table 3 Comparison between both groups regarding sleep architecture and breathing events

\begin{tabular}{llll}
\hline Variable & Group I & Group I & P value \\
& Mean \pm SD & & \\
\hline AHI & $1.44 \pm 3.5$ & $2.58 \pm 5.1$ & 0.315 \\
N1, 2 (min) & $53.2 \pm 23.6$ & $71.15 \pm 22.9$ & 0.004 \\
N3 (min) & $2.23 \pm 3.5$ & $2.99 \pm 6.1$ & 0.552 \\
\hline
\end{tabular}

$\overline{A H I}$ apnea-hypopnea index, O2 oxygen, N1, 2 stage 1,2 sleep, N3 stage 3 sleep
Table 4 Comparison between both groups regarding REM sleep parameters

\begin{tabular}{llll}
\hline Variable & Group I & Group I & $P$ value \\
& Mean \pm SD & & \\
\hline REM (min) & $32.7 \pm 25.4$ & $17.69 \pm 20.2$ & 0.014 \\
REM latency (min) & $87 \pm 56$ & $97.3 \pm 202$ & 0.491 \\
\hline REM rapid eye movement & & &
\end{tabular}

(PHT), lamotrigine (LTG), and oxycarbazepine (OXC) were more in the refractory epilepsy group, yet with no statistical significance (Table 6).

\section{Discussion}

Relationship of sleep and epilepsy is complex and reciprocal. Sleep in epileptic patients is usually altered and this may lead to intractability. Moreover, it is confirmed that prolonged awakening has a negative impact on increased cortical excitability and susceptibility to seizures. In addition, sleep-wake cycle and its regulatory mechanisms can affect epilepsy. Putting these data in mind, we could hypothesize a relation between epilepsy and sleep disorders [8]. In patients with epilepsy, studies of sleep architecture have been a very fruitful area of clinical research [9]. Our study is the first Egyptian study, to our knowledge, to compare between sleep architecture in medically controlled and refractory epilepsy patients.

In this study, we aimed to characterize possible abnormalities in sleep architecture, quality, and distribution of primary sleep disorders in a group of patients with controlled epilepsy versus medically refractory ones. Interestingly, none of the enrolled patients had any seizures during the PSG recordings or the day before. This was a valuable coincidence, since it rules out the possibility of disturbed sleep being attributed directly to epileptic seizures.

Elwan and colleagues (2005) found that untreated epileptic patients had significant excessive daytime sleepiness compared to controls [10]. In the present study, we found that patients with refractory and medically controlled epilepsy reported frequent symptoms of sleep disturbance including insomnia, EDS, and snoring which comes in agreement with a couple of earlier studies [11,

Table 5 Comparison between both groups regarding sleep related clinical data

\begin{tabular}{llll}
\hline Variable & $\begin{array}{l}\text { Group I } \\
\text { Percentage }\end{array}$ & Group II & $P$ value \\
\hline Sleep symptoms & $50 \%$ & $46.7 \%$ & 0.79 \\
Insomnia & $26.7 \%$ & $6.7 \%$ & 0.04 \\
Snoring & $13.3 \%$ & $33.3 \%$ & 0.03 \\
EDS & $10 \%$ & $26.6 \%$ & 0.05 \\
\hline
\end{tabular}

EDS excessive daytime somnolence 
Table 6 Comparison between both groups regarding epilepsy related clinical data

\begin{tabular}{clll}
\hline Variable & Group I & Group I & $P$ \\
& Mean \pm SD or Percentage $(n)$ & value \\
\hline Generalized epilepsy & $83.3 \%(25)$ & $53.3 \%(16)$ & 0.012 \\
Focal epilepsy & $16.7 \%(5)$ & $46.7 \%(14)$ & 0.012 \\
Temporal lobe Seizures & $13.3 \%(4)$ & $43.3 \%(13)$ & 0.009 \\
Number AEDs & $1.03 \pm 0.03$ & $2.6 \pm 1.01$ & 2.71 \\
1st generation AED & $76.7 \%(23)$ & $100 \%(30)$ & 0.005 \\
2nd generation AED & $23.3 \%(7)$ & $90 \%(27)$ & 0.0001 \\
VPA & $30 \%(9)$ & $56.7 \%(17)$ & 0.037 \\
CBZ & $43.3 \%(13)$ & $66.7 \%(20)$ & 0.069 \\
PHT & $3.3 \%(1)$ & $13.3 \%(4)$ & 0.161 \\
LEV & $16.7 \%(5)$ & $60 \%(18)$ & 0.0005 \\
LTG & $3.3 \%(1)$ & $16.7 \%(5)$ & 0.085 \\
OXC & $3.3 \%(1)$ & $6.7 \%(2)$ & 0.55 \\
\hline
\end{tabular}

AEDs antiepileptic drugs, VPA valproic acid, CBZ carbamazepine, $P H T$ phenytoin, LEV levetiracetam, LTG lamotrigine, OXC oxycarbazepine

12]. It was noted that the most frequent sleep disorder among refractory epilepsy patients was snoring followed by EDS. The high prevalence of EDS in the refractory epilepsy group could be attributed to the use of older AEDs and combination of AEDs [3].

However, the most encountered symptom in medically controlled patients was found to be insomnia. This finding was confirmed by the higher mean arousal index, higher limb movement index, and greater sleep fragmentation in the controlled epilepsy group. Vendrame and colleagues found that the prevalence of moderate and severe insomnia was $51 \%$ in their group of epileptic patients, with a stronger association in patients on higher number of AEDs. Also, it should be noted that the prevalence of chronic or severe insomnia in the general population has been estimated to approach $10 \%[13,14]$.

In the present study, the architecture of sleep of medically refractory epilepsy patients was markedly disturbed with greater sleep fragmentation and instability on seizure-free nights compared with medically controlled subjects, and the efficiency of sleep was poorer among the patients with medically refractory epilepsy, which is in accordance with other former studies [11, 15]. These studies agreed that reduction and instability of REM sleep is most often reported, yet shorter total sleep time, lower sleep efficiency, more sleep fragmentation, longer sleep latency, and increased stage shifts and awakenings are also found.

In 40 pediatric-age-group patients, Kaleyias and colleagues reported that intractable epilepsy patients have longer sleep latency, poor sleep efficiency, along with higher arousal index as compared to medically controlled epilepsy [16]. Our findings regarding sleep efficiency, sleep onset latency, REM latency, and AHI are similar to those in the previously mentioned study, which is also in accordance with Zanzmera and colleagues' study. The only major difference observed by us is the much shorter sleep latencies among our patients in both groups, while the intergroup difference noted was similar, which is again similar to the findings reported by Zanzmera and colleagues [15]. It could be attributed to the effect of AEDs, especially CBZ and VPA $[17,18]$.

The interesting finding in our study was that significantly less number of group I patients had PLMs as compared to group II patients. This finding is unlikely to be clinically significant as the index in both groups is quite low, and similar finding was reported before, in the study of Zanzmera and colleagues [15]. This difference could, however, be attributed to AEDs, specifically CBZ and VPA, which might decrease PLMs $[17,19]$.

It is known the AEDs affect sleep structure [20]. Prolonged CBZ and VPA therapy have restorative and normalizing effects on sleep pattern in idiopathic epilepsies, in addition to improving subjective daytime sleepiness. CBZ leads to an increase in the number of sleep stage shifts, a reduction in REM sleep, increased fragmentation of REM sleep, and a significant reduction in sleep latency. In addition, studies suggested that VPA increases the number of arousals, prolongs the light stages of sleep and NREM phase, and decreases the length of the REM phase [21].

In our study, we found that the number of subjects taking CBZ and VPA in group II was much greater than those in group I, which could contribute partially in the observed findings of NREM sleep prolongation and reduction in REM sleep. However, this does not justify the significant decrease in sleep latency in both groups.

\section{Conclusion}

Epilepsy affects sleep architecture and sleep-related events. Patients with refractory epilepsy suffer from more delayed sleep onset latency, REM latency, and respiratory event especially sleep apnea. On the other hand, medically controlled patients suffer from higher arousal index, insomnia, and periodic limb movements. Moreover, antiepileptic drugs, can have a diverse effect on sleep architecture and quality in epileptic patients.

\section{Abbreviations \\ AEDs: Antiepileptic drugs; AHI: Apnea-hypopnea index; BMI: Body mass index; CBZ: Carbamazepine; ECG: Electrocardiogram; EDS: Excessive daytime somnolence; EEG: Electroencephalography; ILAE: International League Against Epilepsy; LEV: Levetiracetam; LTG: Lamotrigine; NREM: Non-rapid eye movement; OXC: Oxycarbazepine; PHT: Phenytoin; PLM: Periodic limb movement; PSG: Polysomnography; REM: Rapid eye movement; VPA: Valproic acid}

\section{Acknowledgements}

Not applicable

Authors' contributions

$\mathrm{OA}$ and HR made substantial contributions to conception and design of the study; HR performed the acquisition of data; MT, LE, NE, NA, and AA 
participated in the analysis and interpretation of data; MT and KA have been involved in drafting the manuscript or revising it critically for important intellectual content; OA has given final approval of the version to be published. All authors agreed to be accountable for all aspects of the work in ensuring that questions related to the accuracy or integrity of any part of the work are appropriately investigated and resolved. All authors read and approved the final manuscript.

\section{Funding}

None

\section{Availability of data and materials}

The datasets generated and analyzed during the current study are not publicly available due to institutional limitations, yet they are available from the corresponding author on reasonable request.

\section{Ethics approval and consent to participate}

This study was approved by the ethical committee of Ain Shams University School of Medicine on the 10th of September 2015. All Subjects provided informed written consent prior to the participating in any study-related activities.

\section{Consent for publication}

Not applicable

\section{Competing interests}

The authors declare that they have no competing interests.

\section{Author details}

${ }^{1}$ Department Neurology and Psychiatry, Faculty of Medicine, Ain Shams University, Cairo, Egypt. ${ }^{2}$ Departemnt of Neurology and Psychiatry, Helwan University, School of Medicine, Helwan, Egypt.

Received: 16 October 2019 Accepted: 19 December 2019

Published online: 07 January 2020

\section{References}

1. Jain SV, Kothare SV. Sleep and epilepsy. Semin Pediatr Neurol. 2015;22(2): 86-92.

2. Kataria L, Vaughn BV. Sleep and epilepsy. Sleep Med Clin. 2016;11(1):25-38

3. Manni R, Tartara A. Evaluation of sleepiness in epilepsy. Clin Neurophysiol. 2000;111(S2):111-4.

4. Kwan P, Brodie MJ. Early identification of refractory epilepsy. New Engl J Med. 2000:342:314-9.

5. Klingmana KJ, Jungquistb CR, Perlis ML. Questionnaires that screen for multiple sleep disorders. Sleep Med Rev. 2017:32:37-44.

6. Berry RB, Brooks R, Gamaldo CE, et al. for the American Academy of Sleep Medicine. The AASM manual for the scoring of sleep and associated events: rules, terminology and technical specifications. Darien: American Academy of Sleep Medicine; 2017.

7. Epstein LJ, Kristo D, Strollo PJ Jr, Friedman N, Malhotra A, Patil SP, et al. Clinical guidelines for the evaluation, management and long-term care of obstructive sleep apnea in adults. J Clin Sleep Med. 2009;5(3):263-76.

8. Bazil CW. Sleep and epilepsy. Semin Neurol. 2017;37(4):407-12.

9. Sudbrack-Oliveira P, Lima Najar L, Foldvary-Shaefer N, da Mota Gomes M. Sleep architecture in adults with epilepsy: a systematic review. Sleep Med. 2019:53:22-7.

10. Elwan H, El-Tamawy M, Saeed S, El-Ghoneimy MT, Shalaby NM, Kishk NA, et al. The Relationship between sleep and epilepsy (A Clinical and Neurophysiological Study). Egypt J Neurol Psychiat Neurosurg. 2005;42(2): 311-22.

11. Li P, Ghadersohi S, Jafari B, Teter B, Sazgar M. Characteristics of refractory vs. medically controlled epilepsy patients with obstructive sleep apnea and their response to CPAP treatment. Seizure. 2012;21(9):717-21.

12. Staniszewska A, Mąka A, Religioni U, Olejniczak D. Sleep disturbances among patients with epilepsy. Neuropsychiatr Dis Treat. 2017;13:1797-803.

13. Vendrame M, Yang B, Jackson S, Auerbach SH. Insomnia and epilepsy: a questionnaire-based study. J Clin Sleep Med. 2013:9(2):141-6.

14. Smith MT, Perlis ML, Park A, Smith MS, Pennington J, Giles DE, et al. Comparative meta-analysis of pharmacotherapy and behavior therapy for persistent insomnia. Am J Psychiatry. 2002;159(1):5-11.
15. Zanzmera P, Shukla G, Gupta A, Singh H, Goyal V, Srivastava A, et al Markedly disturbed sleep in medically refractory compared to controlled epilepsy - a clinical and polysomnography study. Seizure. 2012;21:487-90.

16. Kaleyias J, Cruz M, Goraya JS, Valencia I, Khurana DS, Legido A, et al. Spectrum of polysomnographic abnormalities in children with epilepsy. Pediatr Neurol. 2008;39(3):170-6.

17. Abdulghani MO, Alloush TK, Asaad T, Hemeda M, Salah N, Elsayed L. Effects of antiepileptics therapy on sleep pattern of non-epileptic patients. Egypt J Neurol Psychiat Neurosurg. 2004;41(2):433-42.

18. Vaughn BV, Ali I. Sleep and epilepsy opportunities for diagnosis and treatment. Neurol Clin. 2012;30:1249-74.

19. Lesage $S$, Hening WA. The restless legs syndrome and periodic limb movement disorder: a review of management. Semin Neurol. 2004;24(3): 249-59.

20. Shvarts V, Chung S. Epilepsy, antiseizure therapy, and sleep cycle parameters. Epilepsy Res Treat. 2013:670682.

21. Placidi F, Diomedi M, Scalise A, Marciani MG, Romigi A, Gigli GL. Effect of anticonvulsants on nocturnal sleep in epilepsy. Neurology. 2000;54(5 suppl 1):S25-32.

\section{Publisher's Note}

Springer Nature remains neutral with regard to jurisdictional claims in published maps and institutional affiliations.

\section{Submit your manuscript to a SpringerOpen ${ }^{\circ}$ journal and benefit from:}

- Convenient online submission

- Rigorous peer review

- Open access: articles freely available online

- High visibility within the field

- Retaining the copyright to your article

Submit your next manuscript at $\boldsymbol{\nabla}$ springeropen.com 